Br Heart F 1985; 54: 17-21

\title{
Effect of xamoterol (ICI 118587), a new beta 1 adrenoceptor partial agonist, on resting haemodynamic variables and exercise tolerance in patients with left ventricular dysfunction
}

\author{
ADENIYI O MOLAJO, DAVID H BENNETT \\ From the Regional Cardiac Centre, Wythenshawe Hospital, Manchester
}

SUMMARY The effect of xamoterol, a beta ${ }_{1}$ adrenoceptor partial agonist, on resting haemodynamic measurements and exercise tolerance was studied in 10 patients with dyspnoea of effort. All patients had poor left ventricular function due to myocardial infarction with ejection fractions ranging from $15 \%$ to $35 \%$ (mean $28 \%$ ). The cardiac index and stroke work index both rose significantly. The mean pulmonary artery pressure fell from $20(2) \mathrm{mm} \mathrm{Hg}$ to $16(2) \mathrm{mm} \mathrm{Hg}$ and pulmonary artery wedge pressure from $14(2) \mathrm{mm} \mathrm{Hg}$ to $10(2) \mathrm{mm} \mathrm{Hg}$ within the first four hours. Exercise tolerance, measured on the treadmill, increased significantly in seven patients but was unchanged in the three who had the lowest left ventricular ejection fractions. Exercise heart rate response was attenuated by the drug in all patients.

It is concluded that xamoterol may be beneficial in patients with poor left ventricular function but can be harmful in extremely poor left ventricular function where high sympathetic drive may be important.

Xamoterol $\quad\{(+/-)-1-(4-h y d r o x y p h e n o x y)-3-[2-$ (4-morpholine carbonamido)ethylamino]-propan-2-ol fumarate)\} has been shown in animal experiments to be a beta ${ }_{1}$ adrenoceptor partial agonist with little effect on arterial impedance. ${ }^{1}$ Its partial agonist or intrinsic sympathomimetic activity amounts (at its maximum) to $45 \%$ of the maximum activity of the full agonist isoprenaline. ${ }^{2}$ Previous studies in volunteers showed that at rest it has positive inotropic properties as indicated by shortening of the systolic time intervals and rise in systolic blood pressure. ${ }^{3}$ It attenuates the chronotropic response to exercise. ${ }^{45}$ The beneficial haemodynamic effect of xamoterol in patients at rest with left ventricular dysfunction has been reported recently. ${ }^{6}$ Nevertheless, there is no information about the effect of this drug on exercise tolerance, which is a measurement of practical importance.

We studied the effect of xamoterol on resting haemodynamic variables and exercise tolerance in patients with exertional dyspnoea caused by angio-

Requests for reprints to $\operatorname{Dr}$ A O Molajo, Regional Cardiac Centre, Wythenshawe Hospital, Southmoor Road, Wythenshawe, Manchester M23 9LT.

Accepted for publication 12 February 1985 graphically confirmed myocardial infarction. The study was single blind; xamoterol was given for two weeks followed by placebo for two weeks and finally xamoterol for four weeks. Effort tolerance was measured before treatment and fortnightly thereafter. Haemodynamic measurements were made before and after the first dose of xamoterol and after eight weeks' treatment with xamoterol.

\section{Patients and methods}

PATIENT POPULATION

Ten patients (nine men and one woman), whose mean (SD) age was $54(2.4)$ years, were studied. They all had chronic left ventricular dysfunction and dyspnoea of effort (New York Heart Association (classes II and III). Their mean angiographic left ventricular ejection fraction was $28 \%$ (range $15-35 \%$ ). Two patients (cases 6 and 8) also had moderately severe mitral regurgitation. Table 1 shows the patients' characteristics and treatment at the start of the study. These treatments had failed to improve their symptoms. Vasodilative and antiarrhythmic agents were withdrawn at least two weeks before the start of the study. A prestudy exercise test was performed to determine a suitable exercise protocol for each patient. As a result either 
Table 1 Patient characteristics and treatment at the start of the study

\begin{tabular}{llll}
\hline Case No & LVEF (\%) & Coronary angiography & Medical treament (mg daily) \\
\hline 1 & 15 & LAD blocked, Cx and RCA stenoses & Frusemide 120 \\
2 & 22 & LAD blocked, Cx and RCA stenoses & Frusemide 80; amiloride 10 \\
3 & 32 & LAD and Cx stenoses, RCA blocked & Frusemide 80 \\
4 & 32 & LAD and Cx stenoses, RCA blocked & Frusemide 80; bendrofluazide 5 \\
5 & 35 & LAD and Cx stenosis, RCA blocked & Frusemide 80 \\
6 & 30 & LAD, Cx, and RCA stenoses & Frusemide 120; lanoxin 0.25; \\
7 & 26 & LAD blocked, RCA and Cx stenoses & amiloride 10 \\
8 & 33 & LAD, RCA, and Cx stenoses & Frusemide 80 \\
9 & 18 & LAD blocked, RCA stenosis spironolactone 200 \\
10 & 35 & RCA blocked, LAD and Cx stenoses & Frusemide 80 \\
\hline
\end{tabular}

LVEF, left ventricular angiographic ejection fraction; LAD, left anterior descending; RCA, right coronary artery; Cx, circumflex coronary artery.

the standard or modified Bruce protocol ${ }^{7}$ was chosen for each patient.

\section{EXERCISE TEST PROCEDURE}

Exercise tolerance was assessed approximately 24 hours before the haemodynamic study using the Marquette Case TM Computer Assisted System for Exercise (Marquette Electronics Inc, Milwaukee, USA) using the standard Bruce or a modified Bruce protocol. A maximal symptom limited exercise test was performed. Exercise performance was calculated as the sum of work done during each stage of the Bruce protocol (Fig.1). Work done during each stage of this exercise protocol may be expressed as follows: work done equals $\mathrm{Mg}(\sin \theta+\cos \theta)(\mathrm{S})(\mathrm{T})$ in Joules, where $\theta$ is the angle of inclination of the treadmill; $S$ the speed of the treadmill in $\mathrm{m} / \mathrm{s}$; $T$ the time spent on each stage of exercise in seconds; $g$ the gravitational force $\left(9.8 \mathrm{~m} / \mathrm{s}^{2}\right)$; and $M$ the mass of the patient in $\mathrm{kg}$. Modified lead V1, V5, and aVF electrocardiograms were recorded continuously during the test.

\section{RESTING HAEMODYNAMIC MEASUREMENTS}

Early-On the first day of the study right heart catheterisation was performed at rest, with the patient

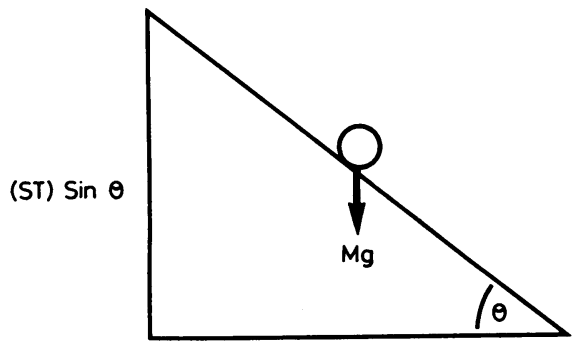

(ST) $\operatorname{Cos} \theta$

Fig. 1 Exercise performance calculated as sum of work done $(m g)$ during each stage of protocol: $M g(\sin \theta+\cos \theta)(S)(T)$ (Foules), where $\theta$, angle of inclination (of treadmill); $S$, speed (of treadmill) $m / s) ; T$, time spent on each stage of exercise (s); $g$, gravitational force $\left(9.8 \mathrm{~m} / \mathrm{s}^{2}\right) ; M$, mass of patient $(\mathrm{kg})$. supine and without premedication, using a flow directed thermodilution catheter. Baseline measurements of pulmonary artery systolic and diastolic pressure, mean pulmonary artery pressure, mean pulmonary capillary wedge pressure, and heart rate were recorded. Cardiac output was measured in triplicate using a Gould Cardiac Index Computer Model SP1435 (Gould Inc., Oxnard, USA). Systemic arterial pressure was measured by a standard sphyg momanometer. Electrocardiographic monitoring was maintained throughout the study. After the baseline haemodynamic variables were measured xamoterol $200 \mathrm{mg}$ was given orally and the measurements repeated $1,2,4,6$, and 12 hours later.

Late-During the eighth week of the trial right heart catheterisation was repeated at rest with the patient supine, without premedication, and before the morning dose of xamoterol. After the baseline haemodynamic variables were measured xamoterol $200 \mathrm{mg}$ was given orally and the measurements repeated one and two hours later.

\section{OUTPATIENT TREATMENT}

Outpatient treatment was continued with xamoterol orally $200 \mathrm{mg}$ twice daily. Clinical examination and exercise tolerance testing were repeated at fortnightly intervals. During the third and fourth weeks patients were changed to treatment with the placebo.

\section{DERIVED HAEMODYNAMIC VARIABLES}

Mean systemic arterial blood pressure (MAP) was calculated as: diastolic pressure plus 1/3 (systolic minus diastolic pressures) in $\mathrm{mm} \mathrm{Hg}$; body surface area in $\mathrm{m}^{2}$ was determined from standard tables from their height and weight; cardiac index (CI) was calculated as $\mathrm{CO} / \mathrm{BSA}\left(1 / \mathrm{min}\right.$ per $\left.\mathrm{m}^{2}\right)$, where $\mathrm{CO}$ is cardiac output $(1 / \mathrm{min})$; stroke volume index (SVI) as: $(\mathrm{CO} \times$ 1000)/BSA $\times \mathrm{HR})\left(\mathrm{ml} / \mathrm{beat} / \mathrm{m}^{2}\right)$, where HR is heart rate (beats/min); left ventricular stroke work index (LVSWI) as: (MAP-PCWP) $\times$ SVI $\times 0.0136(\mathrm{~g} \mathrm{~m} /$ $\mathrm{m}^{2}$ ), where PCWP is pulmonary capillary wedge pressure; and systemic vascular resistance (SVR) as: MAP 
Table 2 Haemodynamic indices in 10 patients before and after a single oral dose of xamoterol $200 \mathrm{mg}$. Values are mean (SEM)

\begin{tabular}{|c|c|c|c|c|}
\hline Haemodynamic indices & Pretreatment value & Peak value & $\begin{array}{l}\text { Time of peak } \\
\text { response (h) }\end{array}$ & p value \\
\hline $\begin{array}{l}\text { Mean systemic arterial pressure }(\mathrm{mm} \mathrm{Hg}) \\
\text { Heart rate (beats/min) } \\
\text { Pulmonary capillary wedge pressure }(\mathrm{mm} \mathrm{Hg}) \\
\text { Mean pulmonary artery pressure }(\mathrm{mm} \mathrm{Hg}) \\
\text { Cardiac index }\left(\mathrm{l} / \mathrm{min} \text { per } \mathrm{m}^{2}\right) \\
\text { Stroke volume index }\left(\mathrm{ml} / \mathrm{beat}^{2} / \mathrm{m}^{2}\right) \\
\text { Systemic vascular resistance }(\text { dyn } \mathrm{s} \mathrm{cm}-\mathrm{s}) \\
\text { Left ventricular stroke work index }\left(\mathrm{g} \mathrm{m} / \mathrm{m}^{2}\right)\end{array}$ & $\begin{array}{l}91(4) \\
69(6) \\
14(2) \\
20(2) \\
2 \cdot 3(0.1) \\
40(3) \\
1693(59) \\
45(4)\end{array}$ & $\begin{array}{l}88(4) \\
76(5) \\
10(2) \\
16(2) \\
3 \cdot 2(0 \cdot 2) \\
46(3) \\
1247(124) \\
53(5)\end{array}$ & $\begin{array}{l}4 \\
4 \\
1 \\
4 \\
4 \\
4 \\
4 \\
4\end{array}$ & $\begin{array}{l}\text { NS } \\
\text { NS } \\
<0.01 \\
<0.001 \\
<0.001 \\
<0.0005 \\
<0.01 \\
<0.0025\end{array}$ \\
\hline
\end{tabular}

$\times$ 80)/CO (dyn s $\mathrm{cm}^{-5}$. All results are expressed as mean (standard error of mean (SEM)) and analysed using Student's paired $t$ test.

\section{Results}

\section{RESTING HAEMODYNAMIC VARIABLES}

On administration of the drug there was an increase in cardiac index and a fall in pulmonary artery pressure. Maximum change in the haemodynamic variables occurred within four hours of giving xamoterol (Table 2 and Figs. 2-4). The drug increased both cardiac index and stroke volume index from $2 \cdot 3(0.1)$ to $3.2(0.2) \mathrm{l} / \mathrm{min}$ per $\mathrm{m}^{2}(\mathrm{p}<0.001)$ and from $40(3)$ to 46 (3) $\mathrm{ml} /$ beat $/ \mathrm{m}^{2}(\mathrm{p}<0.0005)$ respectively (Fig. $4 a$ and $b$ ). Left ventricular stroke work index (Fig. $4 d$ ) was increased from $45(4)$ to $53(5) \mathrm{g} \mathrm{m} / \mathrm{m}^{2}$ ( $\mathrm{p}<0.0025$ ) while systemic vascular resistance fell from 1693(59) to $1247(124)$ dyn s cm$~^{-5}(\mathrm{p}<0.01)$ (Fig. $4 c$ ).
The maximum change in mean pulmonary artery pressure from 20(2) $\mathrm{mm} \mathrm{Hg}$ to 16 (2) $\mathrm{mm} \mathrm{Hg}$ occurred at four hours $(p<0.001)$ and in pulmonary capillary wedge pressure from 14(2) $\mathrm{mm} \mathrm{Hg}$ to $10(2) \mathrm{mm} \mathrm{Hg}$ at one hour $(p<0.01)$ (Table 2, Fig. 2).

Heart rate was not significantly altered in the group as a whole, the control heart rate being $69(6)$ beats/ min and 76(5) at four hours (Table 2). Nevertheless, in the seven patients who subsequently showed an improvement in exercise tolerance the heart rate rose from $69(6)$ to $80(5)$ beats/min at four hours $(p<0.05)$ (Fig. $3 a$ ).

\section{EXERCISE TESTING}

All patients had appreciably impaired exercise tolerance before treatment (Fig. 5, Table 3). After two weeks' treatment with xamoterol there was a significant improvement in exercise tolerance in seven patients $(p<0.05)$ (Fig. 3). One patient failed to show
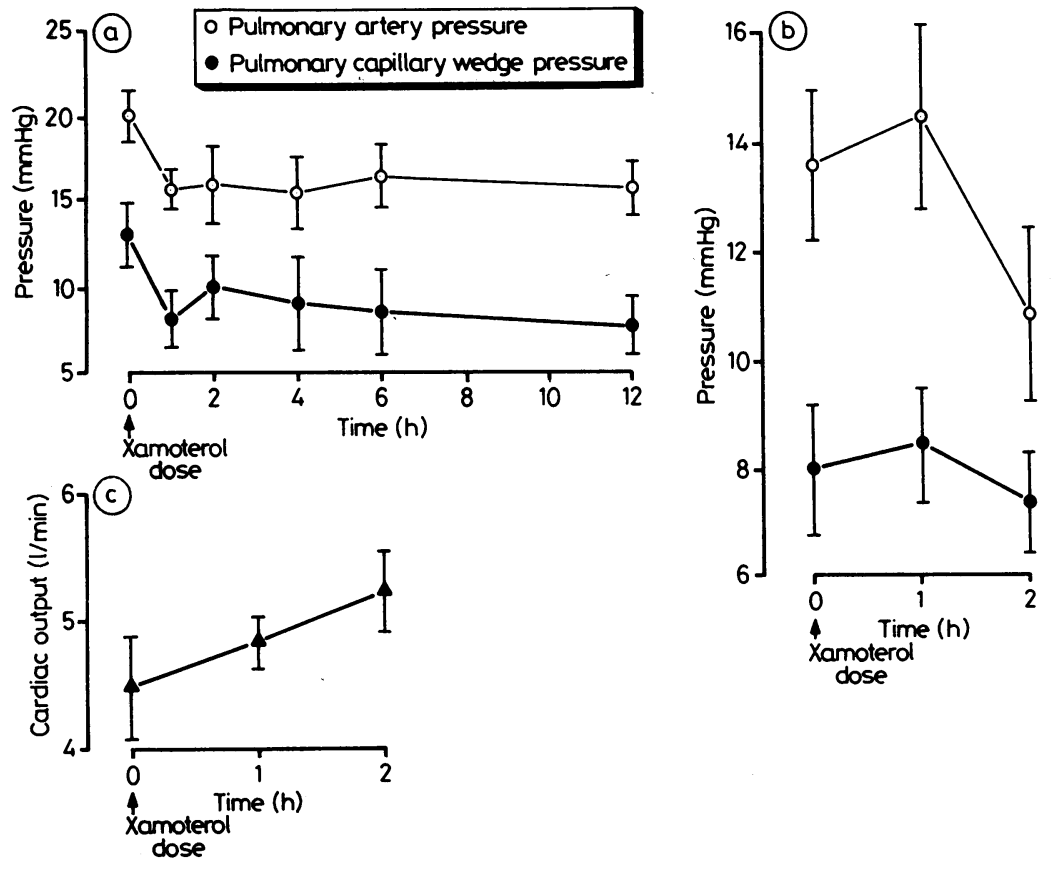

Fig. 2 Resting haemodynamic variables after a single oral dose of xamoterol on (a) the first day and (b) in the eighth week; $(c)$ resting cardiac index after a single oral dose of xamoterol in the eighth week. Values are mean (SEM). 


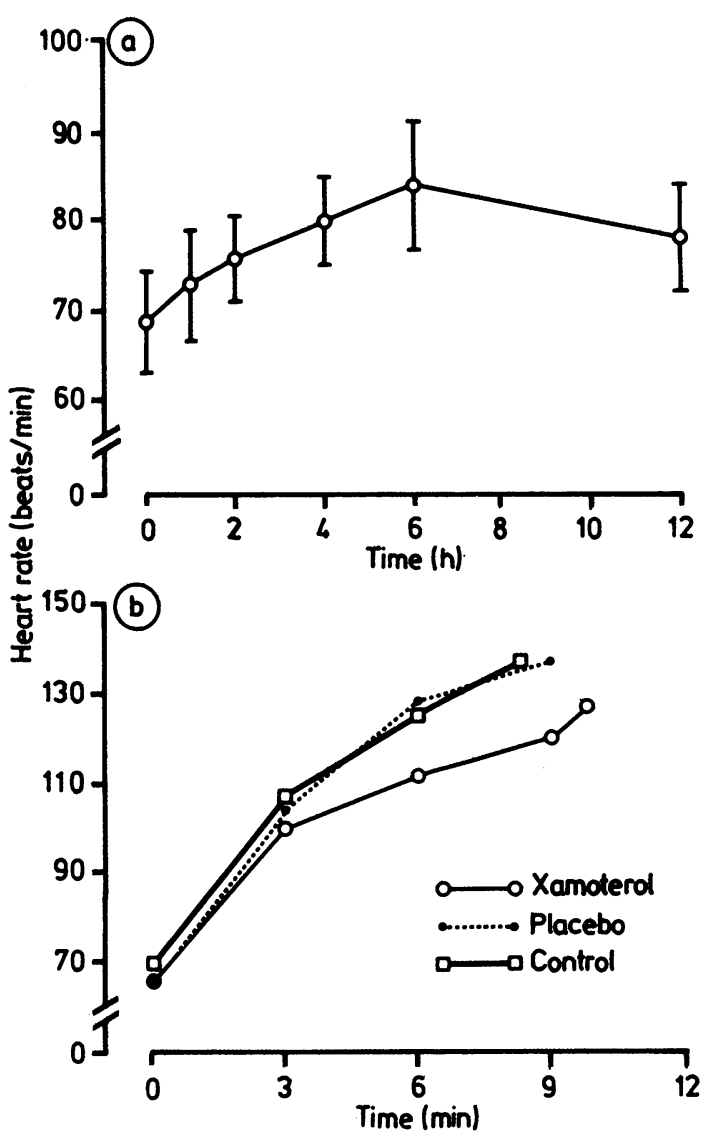

Fig. 3 Heart rate response to a single oral dose of $x$ amoterol $(a)$ at rest and (b) during exercise. Values are mean (SEM).

an improvement in exercise tolerance. Two had stopped treatment before two weeks; one because of nausea and the other on his own initiative because of lack of subjective improvement in symptoms. With placebo the exercise tolerance fell to pretreatment levels in six patients whose exercise tolerance had improved on the drug. It was unchanged in two patients. When xamoterol was restarted there was an improvement in exercise tolerance in the six patients who had deteriorated with placebo and in one of the patients who showed no change in exercise tolerance with placebo. The condition of the other patient who showed no change with placebo deteriorated and he was withdrawn from the study after a further two weeks of xamoterol.

The change in exercise tolerance with the drug was significantly greater than that with placebo $(p<0.05)$ (Table 3). The increase in heart rate with xamoterol after maximal exercise was significantly lower than that with placebo $(\mathrm{p}<0.05)$ (Fig. $3 b)$. (a)

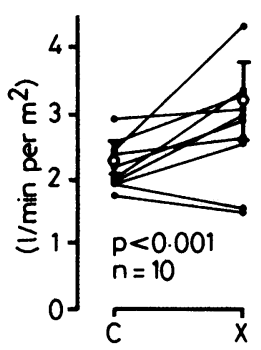

(c) 240

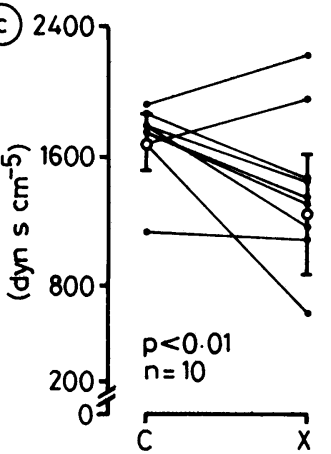

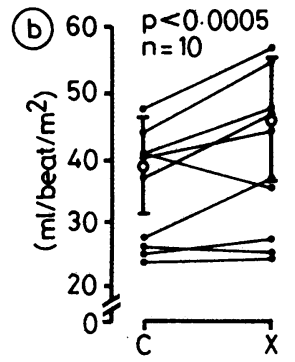

(d)

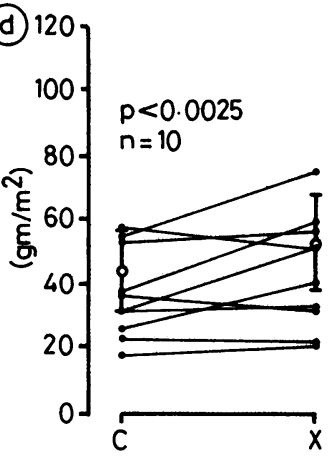

Fig. 4 Haemodynamic variables before $(C)$ and after $(X)$ peak response to a single oral dose of xamoterol: (a) cardiac index; $(b)$ stroke vohome index; (c) systemic vascular resistance; and (d) left ventricular stroke work index.

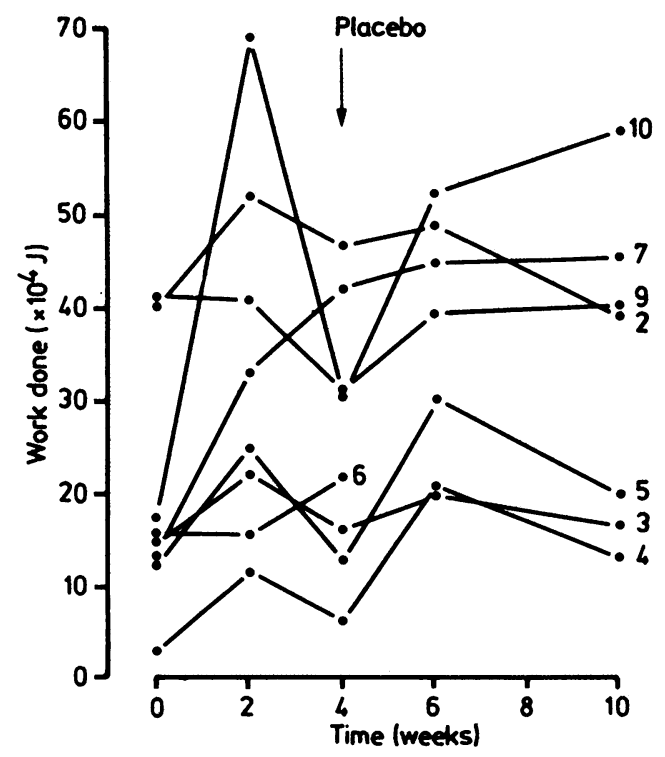

Fig. 5 Changes in exercise tolerance in the 10 patients during long term administration of xamoterol and placebo. Numbers are case numbers. 
Table 3 Exercise tolerance in study patients. Values are mean (SEM)

\begin{tabular}{|c|c|c|c|}
\hline Time (weeks) & Treatment given & Work done $\left(7 \times 10^{4}\right)$ & $p$ value $(n)^{\star}$ \\
\hline $\begin{array}{l}0 \\
0-2 \\
2-4 \\
4-6 \\
6-10\end{array}$ & $\begin{array}{l}\text { Baseline } \\
\text { Xamoterol } 200 \mathrm{mg} \text { twice daily } \\
\text { Placebo } \\
\text { Xamoterol } 200 \mathrm{mg} \text { twice daily } \\
\text { Xamoterol } 200 \mathrm{mg} \text { twice daily }\end{array}$ & $\begin{array}{l}20.0(4 \cdot 8) \\
34 \cdot 1(6 \cdot 9) \\
26 \cdot 7(5 \cdot 2) \\
39 \cdot 7(4 \cdot 9) \\
33 \cdot 6(6 \cdot 5)\end{array}$ & $\begin{array}{l}(10) \\
<0.05(8) \\
(8) \\
<0.05(7) \\
<0.05(7)\end{array}$ \\
\hline
\end{tabular}

^Significance levels derived from comparison of exercise tolerance with placebo and xamoterol.

\section{Discussion}

Previous work suggests that there is increased activity of the sympathetic system as well as changes in the reninangiotensin and vasopressin neurohumoral systems in chronic heart failure. ${ }^{8}$ These pathophysiological derangements occur to varying degrees in different patients. Xamoterol has been shown to have $40 \%$ of the chronotropic and inotropic effects of the pure agonist isoprenaline. ${ }^{2}$ During exercise it reduces the chronotropic response to sympathetic stimulation. ${ }^{49}$ It therefore shows both beta adrenoceptor agonism and antagonism, depending on the prevailing level of sympathetic activity; it is a beta ${ }_{1}$ adrenoceptor partial agonist.

This study shows that xamoterol can improve resting haemodynamic variables and exercise tolerance in some patients with pronounced impairment of left ventricular function caused by myocardial infarction. At low levels of sympathetic activity it acts as a beta ${ }_{1}$ adrenoceptor agonist as shown by the increase in stroke volume, left ventricular stroke work index, and heart rate and the fall in pulmonary capillary wedge pressure that occurred in most patients. The fall in systemic vascular resistance is probably the result of increased cardiac output promoting a reduction in endogenous sympathetic activity rather than a direct vasodilator action. Certainly, work in animals has shown that the drug is not a vasodilator. It is likely that in the two patients in whom the drug did not have a positive inotropic or positive chronotropic effect sympathetic tone was higher and the drug acted as a beta adrenoceptor antagonist. These two patients had the lowest ejection fractions of the group.

The patients in whom the drug caused a positive chronotropic effect at rest experienced an improvement in exercise tolerance, whereas those in whom the drug had a negative chronotropic effect and who presumably had higher levels of sympathetic activity at rest did not benefit in terms of exercise tolerance.

The results from this small study suggest that whereas the drug can be given safely and with benefit to patients with poor left ventricular function (mean ejection fraction $28 \%$ ) it may be harmful to patients with extremely poor ventricular function who depend on high levels of sympathetic nervous system activity.

We thank Mr Eric B Faragher for statistical assistance.

\section{References}

1 Barlow JJ, Main BG, Snow HM. Beta-adrenoceptor stimulant properties of amidoalkylamino-substituted 1-aryl-2-ethanols and 1-(aryloxy)-2-propanols. $f$ Med Chem 1981; 24: 315-22.

2 Nuttall A, Snow HM. The cardiovascular effects of ICI 118587: a beta ${ }_{1}$ adrenoceptor partial agonist. $\mathrm{Br} \mathcal{F}$ Pharmacol 1982; 77: 381-8.

3 Marlow HF, Harry JD, Shields AG. Duration of action of single intravenous doses of ICI 118587 a cardiac betastimulant. First World Conference on Clinical Pharmacology and Therapeutics, London. Abstract No 0772, 1980.

4 Harry JD, Marlow HF, Wardleworth AG, Young J. The action of ICI 118587 (a beta-adrenoceptor partial agonist) on the heart rate response to exercise in man [Abstract]. Br f Clin Pharmacol 1981; 12: 266P-7P.

5 Barlow JJ, Main BG, Nuttall A, Moors J, Snow HM. The cardiovascular activity of ICI 118,587 , a novel betaadrenoceptor partial agonist [Abstract]. $\mathrm{Br} \mathcal{F}$ Pharmacol 1979; 67: 412 .

6 Rousseau MF, Pouleur H, Vincent MF. Effects of a cardioselective beta 1 partial agonist (Corwin) on left ventricular function and myocardial metabolism in patients with previous myocardial infarction. Am $\mathcal{J}$ Cardiol 1983; 51: 1267-74.

7 Bruce RA; Hornsten TR. Exercise stress testing in evaluation of patients with ischemic heart disease. Prog Cardiovase Dis 1969; 11: 371-90.

8 Cohn JN, Levine TB, Francis GS, Goldsmith S. Neurohumoral control mechanisms in congestive heart failure. Am Heart f 1981; 102: 509-14.

9 Molajo AO, Coupe MO, Bennett DH. Effect of Corwin (ICI 118587) on resting and exercise heart rate and exercise tolerance in digitalised patients with chronic atrial fibrillation. Br Heart $\mathcal{F}$ 1984; 52: 392-5. 